\title{
The geometry of an electron scale magnetic cavity in the plasma sheet
}

\author{
H. Liu ${ }^{1,2}$, Q.-G. Zong ${ }^{1}$, H. Zhang ${ }^{2}$, W. J. Sun ${ }^{3}$, X.-Z. Zhou ${ }^{1}$, Daniel J. \\ Gershman $^{4}$, C. Shi ${ }^{5}$, K. Zhang ${ }^{6,7}$, Guan Le ${ }^{4}$, C. Pollock ${ }^{8}$ \\ ${ }^{1}$ Institute of Space Physics and Applied Technology, Peking University, Beijing 100871, China. \\ ${ }^{2}$ Geophysical Institute, University of Alaska Fairbanks, Fairbanks, AK 99775, USA. \\ ${ }^{3}$ Department of Climate and Space Sciences and Engineering, University of Michigan, Ann Arbor, MI \\ 48109, USA. \\ ${ }^{4}$ NASA, Goddard Space Flight Center, Greenbelt, MD 20771, USA. \\ ${ }^{5}$ Department of Earth, Planetary, and Space Sciences, University of California, Los Angeles, CA 90095, \\ USA. \\ ${ }^{6}$ Laboratory for Atmospheric and Space Physics, University of Colorado, Boulder, Colorado 80303, USA. \\ ${ }^{7}$ Ann and H. J. Smead Department of Aerospace Engineering Sciences, University of Colorado, Boulder, \\ Colorado 80309, USA. \\ ${ }^{8}$ Denali Scientific, 3771 Mariposa Lane, Fairbanks, AK 99709, USA.
}

\section{Key Points:}

- A comprehensive energetic particle sounding technique is applied to detect the geometry of an electron scale magnetic cavity.

- The boundary loss plays a significant role in forming electron non-gyrotropic distributions in the cavity.

- Moving speed obtained by the technique is higher for the trailing boundary than the leading boundary suggesting a shrinkage of the cavity.

This is the author manuscript accepted for publication and has undergone full peer review but has not been through the copyediting, typesetting, pagination and proofreading process, which may lead to differences between this version and the Version of Record. Please cite this article as doi: 10.1029/2019GL083569

Corresponding author: Q.-G. Zong, qgzong@pku.edu.cn 


\section{0

\begin{abstract}
Electron scale magnetic cavities are electron vortex structures formed in turbulent plasma, while the evolution and electron dynamics of these structures have not been fully understood. Recently, high energy, angular, and temporal electron measurements from MMS have enabled the application of an energetic particle sounding technique to these structures. This study analyzes an electron scale magnetic cavity observed by MMS on 7 May 2015 in the plasma sheet. A comprehensive sounding technique is applied to obtain the geometry and propagation velocities of the boundaries. The result shows that the scale size of the structure is $\sim 90 \mathrm{~km}$, and the leading and trailing boundaries are moving in the same direction but with different speeds $(\sim 11.5 \pm 2.2 \mathrm{~km} / \mathrm{s}$ and $\sim 18.1 \pm 3.4 \mathrm{~km} / \mathrm{s}$, respectively). The speed difference suggests a shrinking of the structure that may play a significant role in magnetic energy dissipation and electron energization of electron scale magnetic cavities.
\end{abstract}

\title{
Plain Language Summary
}

Electron scale magnetic cavities are extremely small vortices frequently found in space plasma environments, where a cavity of the depressed magnetic field contains energetic electrons forming a ring-like current at electron scale. The formation of these structures is thought to be linked to energy cascade in plasma turbulence, while the mechanism and process of the energy transformation is not clear. In this paper we discuss an electron scale magnetic cavity in the terrestrial plasma sheet with a modified particle sounding technique proposed and applied to the boundaries, suggesting that the structure is shrinking. This analysis may provide new insight into understanding the evolution and electron energization of these structures.

\section{Introduction}

Electron scale magnetic cavities, commonly referred to as electron scale magnetic holes, are widely observed in the magnetotail plasma sheet (Gershman et al., 2016; Goodrich, Ergun, \& Stawarz, 2016; Goodrich, Ergun, Wilder, et al., 2016; Sun et al., 2012; S. T. Yao et al., 2016; Zhang, Artemyev, Angelopoulos, \& Horne, 2017) and the magnetosheath (Huang, Du, et al., 2017; Huang, Sahraoui, et al., 2017; S. Yao et al., 2017). They are thought to be sheet-like or cylindrical structures with scales less than proton thermal gyro-radii (Ji et al., 2014; Li et al., 2016; Liu et al., 2019; Sundberg, Burgess, \& Haynes, 2015). Recent observations and simulations have shown that they are characterized by electron vortex and may facilitate generations of various kinds of waves (Huang et al., 2019; S. T. Yao et al., 2019), and are also possibly linked to energy cascade in turbulent plasma (Haynes, Burgess, Camporeale, \& Sundberg, 2015; Roytershteyn, Karimabadi, \& Roberts, 2015; Sahraoui et al., 2004, 2006). However, the electron dynamics and generating mechanisms are still not fully settled.

The energetic particle sounding technique is a remote sensing method that is able to draw a dynamic view of a trapping boundary based on particle distribution function measurements (Kaufmann \& Konradi, 1973; Konradi \& Kaufmann, 1965). In the past, the sounding technique has been successfully applied to proton distributions to sense large scale structures such as the magnetopause (e.g., Oksavik, Fritz, Zong, Søraas, \& Wilken, 2002; Zong et al., 2004), while the application to electron data is only made possible by the launch of Magnetospheric Multiscale (MMS) mission in March 2015 (Burch, Moore, Torbert, \& Giles, 2016), demonstrated by Liu et al. (2019). The Fast Plasma Investigation (FPI) instruments (Pollock et al., 2016) onboard MMS are able to provide very high quality phase space density (PSD) measurements, of which the time resolution is up to 30 milliseconds for electrons and 150 milliseconds for protons, and the angular resolution is up to $32 \times 16$, favorable for the sounding technique. 
In this paper, we describe and discuss an electron scale magnetic cavity (ESMC) event observed by MMS on May 7th 2015 in the plasma sheet, which has been previously reported by Gershman et al. (2016). We use burst mode magnetic field data from Fluxgate Magnetometers (FGM) (Russell et al., 2016) and burst mode electron distributions and moments from FPI. In this paper, a comprehensive sounding technique is proposed to obtain the moving velocity of the boundaries, suggesting that the structure is shrinking. Finally, we discuss the significance of the shrinkage in understanding the phenomenon and the potential impact of these structures on tail dynamics.

\section{Method}

\subsection{Energetic particle sounding technique}

This technique assumes a finite Larmor radius effect near a planar trapping boundary. Here a trapping boundary means that a particle will regularly maintain its gyrating motion inside the boundary but could be lost while outside the boundary. The particle loss happened outside is referred to as boundary loss in this paper. For a particle detector close enough to such a boundary (less than twice of the particle gyro-radius), some look directions of the detector would correspond to particle gyro-orbits intersecting with the boundary, leading to significant decreases of particle fluxes in these directions. Thus in the plane perpendicular to the magnetic field, the measured particle distributions would be non-gyrotropic due to the boundary loss, as illustrated by the bottom part of Figure 4 (the same as Figure 1 of Zong et al. (2004) but for GSM coordinates). In that case, two critical look directions $\left(\phi_{A}\right.$ and $\left.\phi_{D}\right)$ corresponding to gyro-orbits tangent to the boundary, recognized by sharp decreases in phase space density, can be utilized to calculate the orientation $(\beta)$ and the distance $(R)$ of the boundary, where the orientation means the normal direction of the planar trapping boundary.

The equations are given by Zong et al. (2004):

$$
\begin{aligned}
& \beta=\frac{\phi_{A}+\phi_{D}}{2}+\frac{\pi}{2} \\
& R=\rho-\rho \cdot \cos \left(\frac{\phi_{D}-\phi_{A}}{2}\right)
\end{aligned}
$$

where $\rho$ is the gyro-radius of the particle.

The uncertainty originates from finite angular resolution $(\Delta \phi)$, finite time resolution $(\Delta t)$, and uncertainty in gyro-radius $(\Delta \rho)$ (Liu et al., 2019; Zong et al., 2004):

$$
\begin{aligned}
& \Delta \beta=\Delta \phi \\
& \Delta R=\sqrt{\left(2 R \rho-R^{2}\right) \Delta \phi^{2}+\left(\frac{R}{\rho} \Delta \rho\right)^{2}+(V \Delta t)^{2}}
\end{aligned}
$$

where $\Delta \rho$ is from finite energy resolution and $V$ is the motion speed of the structure.

\subsection{A comprehensive sounding technique}

For a specific situation that the boundary orientation $\left(\beta_{f}\right)$ does not change during the crossing and the distance increases at a fixed speed $\left(V_{m}\right)$, the sounding technique can be transformed to a data fitting method to directly obtain the propagation velocity. Equations (1) and (2) are rewritten as:

$$
\begin{aligned}
& R=V_{m}\left(t-t_{0}\right) \\
& \phi_{H}=\frac{\phi_{D}-\phi_{A}}{2}=\arccos \left(1-\frac{R}{\rho}\right) \\
& \phi_{A}=\beta_{f}-\frac{\pi}{2}-\phi_{H}
\end{aligned}
$$




$$
\phi_{D}=\beta_{f}-\frac{\pi}{2}+\phi_{H}
$$

where $t_{0}$ is the time when the spacecraft crossed the boundary (i.e. $R=0$ ).

Equations (5) - (8) indicate that $\phi_{A}$ and $\phi_{D}$ can be functions of time controlled by three global coefficients $\beta_{f}, V_{m}$, and $t_{0}$. In the plane perpendicular to the magnetic field, critical look directions are expressed by gyro-phase angles $(\phi)$, thus Equations (5) - (8) can be further rewritten as a sinusoidal profile:

$$
t=t_{0}+\frac{\rho}{V_{m}}\left[\sin \left(\phi-\beta_{f}\right)+1\right] \equiv f_{t}\left(\phi \mid \beta_{f}, V_{m}, t_{0}\right)
$$

which means that $\phi_{A}\left(\right.$ or $\left.\phi_{D}\right)=\phi$ should appear at $t=f_{t}\left(\phi \mid \beta_{f}, V_{m}, t_{0}\right)$.

Since critical look directions are defined as sharp $\mathrm{PSD}_{\perp}$ decreases, Equation (9) actually gives the edge of the enhancement region in gyro-phase distributions (i.e. a $2 \mathrm{D}$ map in time and gyro-phase-angle space for each energy channel as illustrated in panels (b) - (h) of Figure 4). Thus, the enhancement region is $t>f_{t}\left(\phi \mid \beta_{f}, V_{m}, t_{0}\right)$, or we could say that the $2 \mathrm{D}$ gyro-phase distribution model should be:

$$
h^{*}\left(t, \phi \mid \beta_{f}, V_{m}, t_{0}\right)= \begin{cases}1, & t>f_{t}\left(\phi \mid \beta_{f}, V_{m}, t_{0}\right) \\ 0, & t<f_{t}\left(\phi \mid \beta_{f}, V_{m}, t_{0}\right)\end{cases}
$$

and three global coefficients $\beta_{f}, V_{m}$, and $t_{0}$ can be determined by fitting Equation (10) to the measured distributions.

For an actual fitting task we consider using a sigmoid function instead of a step function:

$$
h\left(t, \phi \mid \beta_{f}, V_{m}, t_{0}\right)=\frac{1}{1+\exp \left[-\omega_{\text {sample }}\left(t-f_{t}\left(\phi \mid \beta_{f}, V_{m}, t_{0}\right)\right)\right]}
$$

and a least squares fit is determined by:

$$
\operatorname{minimize} \sum_{\substack{\left(t_{i}, \phi_{i}\right) \\ E_{i}}}\left[h_{i}-h\left(t_{i}, \phi_{i} \mid \beta_{f}, V_{m}, t_{0}\right)\right]^{2}
$$

where $\omega_{\text {sample }}$ is the sample rate, $\left(t_{i}, \phi_{i}\right)$ is a data point in gyro-phase distributions, $h_{i}$ is the observed value, and the subscript $E_{i}$ means a global minimum over a series of energy channels. It is worth mentioning that particle gyro-radius $\rho$ is energy dependent. For the event we discussed, 0.03s resolution FPI data looks chaotic due to insufficient count rates, and only after being accumulated to $0.3 \mathrm{~s}$ time resolution the data shows statistical properties. So all the FPI data used in this paper is accumulated to $0.3 \mathrm{~s}$ resolution by smoothing. Thus here we let $\omega_{\text {sample }}=1 / 0.3$.

This technique is a comprehensive version of the sounding technique that can be applied to a boundary with constant motion. If applicable, the curve fitting directly gives the motion speed of the boundary and evaluate the uncertainty.

\section{Observation}

\subsection{An electron scale magnetic cavity in the plasma sheet}

The ESMC event is characterized by a sudden dip of the magnetic field strength $\left(B_{t}\right)$ simultaneously with an enhancement of electron flux with near $90^{\circ}$ pitch angles, as illustrated in Figure 1. Panel (a) is the ambient magnetic field between 06:00 and 06:30 UT, when MMS3 was located in the magnetotail plasma sheet. The spacecraft was close to the central plasma sheet revealed by a very small $B_{t}(\sim 1.6 \mathrm{nT})$ at about 06:13:30 UT. The ESMC event discussed in this paper was observed by MMS3 at around 06:10:50 UT, 
where a sudden $B_{t}$ dip was marked by green arrows in panel (a). Panels (b) - (f) are observations of the ESMC in electron scales. Panel (b) shows a $B_{t}$ depression of $\sim 8 \%$ (from $17.1 \mathrm{nT}$ to $15.8 \mathrm{nT}$ ) between 06:10:47.6 and 06:10:53.9 UT, while the field direction remains almost unchanged (less than 3 degrees, not shown).

Panels (c) - (e) present electron pitch angle distributions (PADs) at low, middle, and high energies, respectively. It is clear that for the PAD of the middle energy (panel (d)), there is a significant enhancement of electron flux near $90^{\circ}$ pitch angles simultaneously with the $B_{t}$ depression. The magenta lines in the three panels are loss cone angles derived from a local magnetic mirror assumption with the Equation:

$$
\sin \left(\alpha_{l}\right)=\sqrt{\frac{B_{t}}{B_{o}}}
$$

where $B_{t}$ is the observation in panel (b) and $B_{o}$ is the ambient strength $(17.1 \mathrm{nT})$. A good agreement between the assumed mirror trapping region and the enhancement of PAD implies that there are trapped electrons within the structure, while the field-aligned configuration is still not clear.

Here we define a local field-aligned coordinate system for a more clear presentation of observations. As mentioned above, the magnetic direction remains almost unchanged, so a mean magnetic field is used as a constant Z-axis to represent the field-aligned direction. The $\mathrm{Y}$-axis is defined by a two-step process using a minimum variance analysis (MVA) (Sonnerup \& Cahill Jr, 1967). We first apply MVA to the electron mass flow $\left(N_{e} V_{e}\right)$ between 06:10:43 and 06:10:57 UT to get the maximum variance direction, where $N_{e}$ is the electron number density and $V_{e}$ is the electron bulk velocity. We then project the maximum variance direction to the plane perpendicular to the $\mathrm{Z}$-axis to get the $\mathrm{Y}$ direction. The X-axis completes the orthogonal set. The newly defined XYZ coordinates are static in GSE:

$$
\begin{aligned}
& e_{X}=\left[\begin{array}{ll}
-0.42,-0.63,0.65
\end{array}\right] \\
& e_{Y}=\left[\begin{array}{ll}
0.86,-0.03,0.52
\end{array}\right] \\
& e_{Z}=\left[\begin{array}{ll}
-0.30,0.77,0.55
\end{array}\right]
\end{aligned}
$$

The reason the variation of $\left(N_{e} V_{e}\right)$ is emphasized for the new coordinate system (by the application of the MVA method) is that the electron vortices are widely believed to actually support or induce the decrease in magnetic field (e.g., Gershman et al., 2016). Panel (f) of Figure 1 shows clear evidence of electron vortex, in which the $V_{e}$ (0.3s time resolution) is projected into the newly defined XYZ coordinates to exhibit a dominant bipolar variation in Y component.

Figure 2 shows sky-maps of electron PSDs at different times from MMS3. The skymaps are organized in a De-spun Body Spacecraft Coordinate System (DBCS) which is very close to the GSE coordinates (Pollock et al., 2016). In this figure, the data is accumulated to $0.3 \mathrm{~s}$ time resolution due to insufficient count rates, and a certain energy channel is chosen as an example.

Panels (a) - (e) show observations near the leading boundary where the spacecraft entered the ESMC. In panel (a), the spacecraft was located outside the ESMC, and there is no enhancement. Panel (b) is $0.6 \mathrm{~s}$ later when the spacecraft entered the structure but was still very close to the boundary, and the sky-map shows clear enhancement as well as inhomogeneity inside the mirror trapping region (two magenta lines from Equation (13)). In panels (c) - (e) the spacecraft was moving farther away from the boundary, and the enhancement region extended but was still confined in the mirror trapping region. In panel (e) the spacecraft was far enough from the boundary so the non-gyrotropy disappeared. The sky-maps when the spacecraft crossed the trailing boundary are also plotted in panels (f) - (j), showing a similar but reversed process to what described above. 

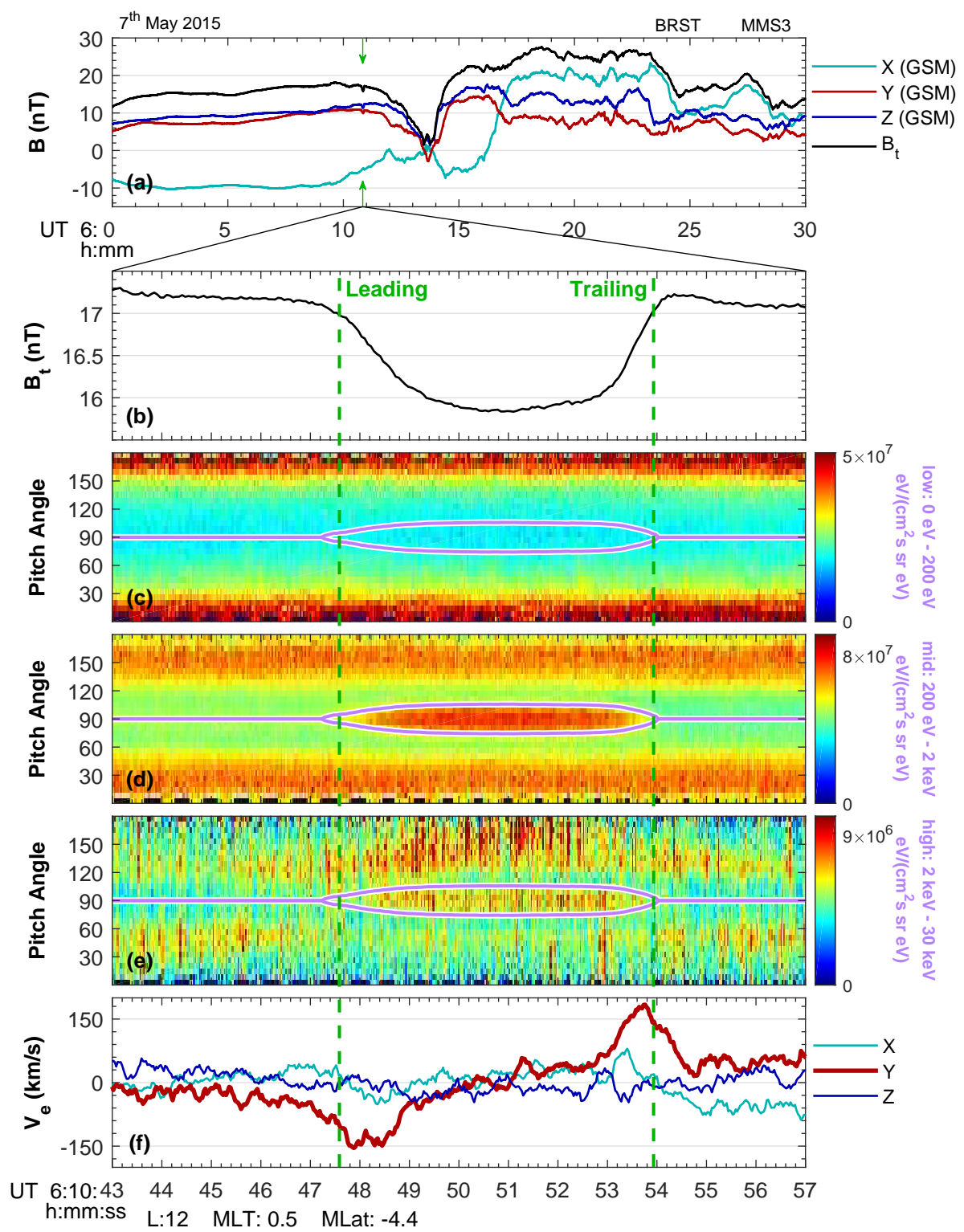

Figure 1. An electron scale magnetic cavity (ESMC) observed in the magnetotail plasma sheet. Panel (a) shows the ambient magnetic field in the GSM coordinates, where a magnetic dip in the total strength $\left(B_{t}\right)$ line, interpreted as an ESMC, is marked by green arrows. Panels (b) (f) further present observations in a very small time scale ( 10s). Panel (b) is the MMS3 observation of $B_{t}$, showing a clear depression between 6:10:47.6 and 6:10:53.9 UT. The electron pitch angle distributions for low, middle, and high energies are plotted in panels (c) - (e), respectively, where the magenta lines are the local loss cone angles $\left(\alpha_{l}\right)$ derived from Equation (13). Panel (f) shows the variation of electron bulk velocity $\left(V_{e}\right)$ in the newly defined XYZ coordinates, detailed in the text. The two vertical dashed lines across panels (b) - (f) are the same as in Figure 4, representing the leading and the trailing boundaries of the structure. The magnetic position is labeled at the very bottom of the figure. 


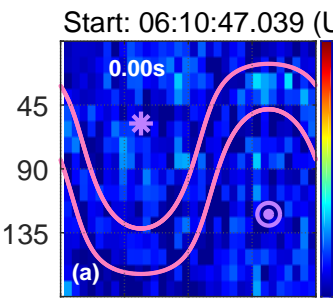

$90 \quad 180270$

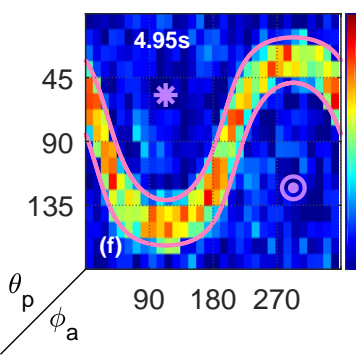

$\mathrm{E}: 842.8-1224.2(\mathrm{eV})$

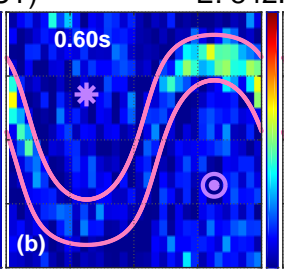

$90 \quad 180 \quad 270$

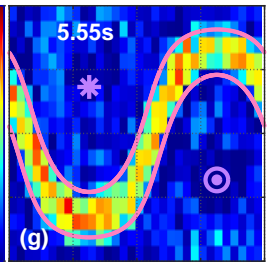

90180270

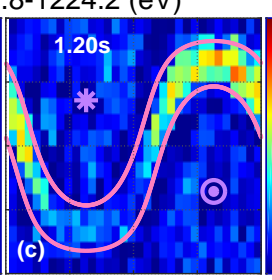

$90 \quad 180270$

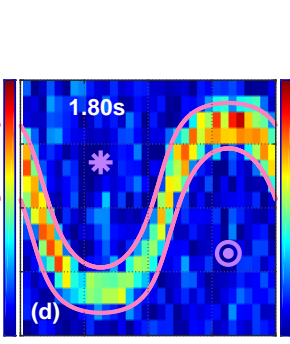

$90 \quad 180270$

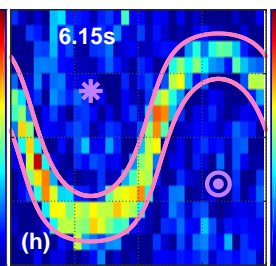

90180270

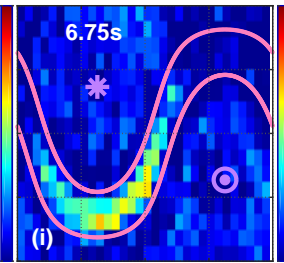

$90 \quad 180 \quad 270$ $\left(10^{-18} \mathrm{~s}^{3} / \mathrm{m}^{6}\right)$

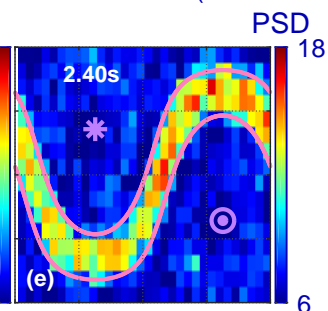

$90 \quad 180270$

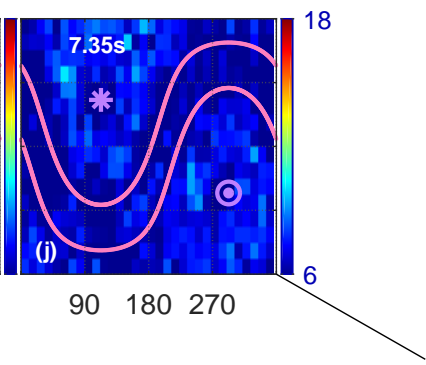

Figure 2. Sky-maps of electron phase space density (PSD) for a certain energy channel observed by MMS3. Panels (a) - (e) are observations near the leading boundary of the ESMC, and panels (f) - (j) are near the trailing boundary. In each panel the horizontal and vertical axes are the azimuthal angles $\left(\phi_{a}\right)$ and the polar angles $\left(\theta_{p}\right)$ in DBCS coordinates, respectively. The asterisk $\left(^{*}\right)$ and the circle $(\odot)$ represent parallel and anti-parallel directions of the magnetic fields. Two magenta lines are the local loss cone angles $\left(\alpha_{l}\right)$ of a magnetic mirror derived from Equation (13). The measuring time is labeled on the top of each panel, starting from 6:10:47.039 UT.

\subsection{The geometry of the structure}

As introduced in the Method section, non-gyrotropic distributions caused by a nearby trapping boundary can be used to remotely detect the boundary by the sounding technique. Figure 2 has illustrated that the non-gyrotropy of $\mathrm{PSD}_{\perp}$ is prominent in the skymaps near the leading and the trailing boundaries, therefore $\phi_{A}$ and $\phi_{D}$ can be identified from data. Then the sounding result, i.e. a combination of $\beta$ and $R$, can be obtained based on Equations (1) and (2).

Figure 3 shows sounding results from multi-energy channels and different times, detailed in the caption. The results are split into two parts corresponding to the leading or the trailing boundaries. In panel (d) the dotted lines at $R=0$ represent sky-maps with no enhancements (like panel (a) of Figure 2). The horizontal dashed lines are from sky-maps with clear but gyrotropic enhancements (like panel (e) of Figure 2), which would occur when the distance is larger than $2 \rho$. The uncertainty $\Delta \beta$ and $\Delta R$ in panels (c) and (d) are calculated from Equations (3) and (4), respectively (for $\Delta R$ an estimation of supremum is used here).

It is shown in panels (c) and (d) that the sounding results from different energy channels are consistent with each other. The distances increase with time near the leading boundary and decrease to zero near the trailing boundary, as expected. In panel (d) the distances exceeding $2 \rho$ happen closer to the boundary at lower energies because $\rho$ is smaller. The orientations in panel (c) indicate that the leading and the trailing boundaries are oriented in anti-parallel directions $\left(\sim 180^{\circ}\right.$ degrees $)$. 


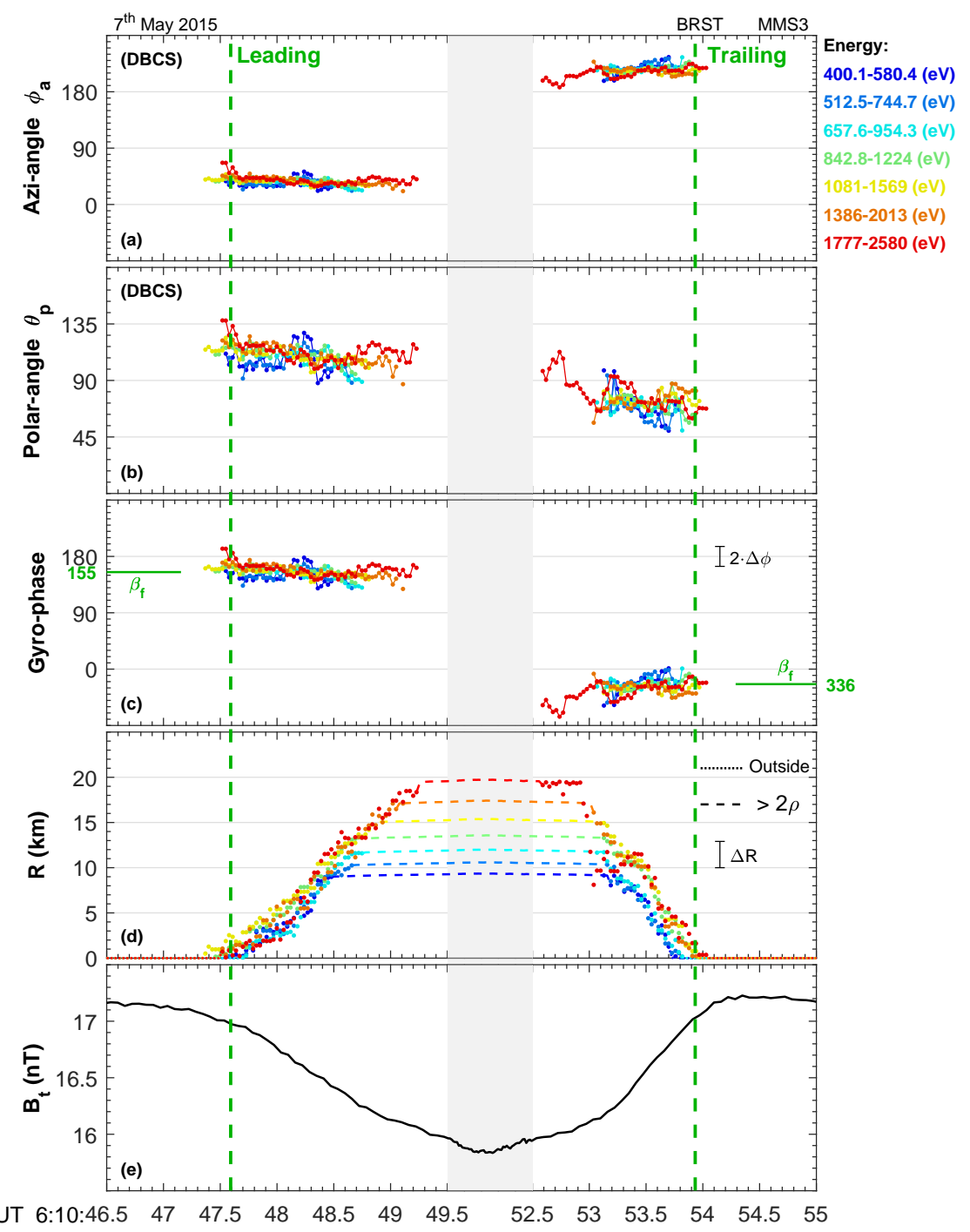

Figure 3. Boundary orientations $(\beta)$ and distances $(R)$ to the spacecraft derived from the energetic particle sounding technique (Equations (1) and (2)). $\beta$ can be expressed as azimuthal (panel (a)) and polar (panel (b)) angles in DBCS coordinates, or gyro-phase angles (panel (c)) in the newly defined XYZ coordinates. The gyro-phase angle is defined as the angle to the positive $\mathrm{X}$-axis in the XY plane. In panel $(\mathrm{d})$ the solid dots are distances $(R)$ between the boundary and the spacecraft; the dotted lines at $R=0$ represent that the spacecraft is located outside the structure; the horizontal dashed lines mean that the spacecraft is inside the ESMC but the distance is larger than twice of the particle gyro-radius $(\rho)$. The variation of $B_{t}$ is shown again in panel (e). Different energies are labeled as different colors. The two vertical dashed lines are the same as in Figure 4, representing the leading and the trailing boundaries. The gray shaded area between 6:10:49.5 and 6:10:52.5 is shrunk to reduce meaningless space in this figure. The orientation $\beta_{f}$, a best fit coefficient shown and discussed mainly in Figure 4, is also labeled in panel (c) of this figure as a horizontal green line for each boundary. 
A constant motion speed of the trapping boundary may make the comprehensive sounding technique applicable to the structure. Figure 4 shows observations of gyro-phase distributions of $\mathrm{PSD}_{\perp}$ and the fitting results. In panels $(\mathrm{b})-(\mathrm{h})$ the electron PSDs with $90^{\circ}$ pitch-angles are extracted from sky-maps to get the gyro-phase distributions (i.e. the $\left.\mathrm{PSD}_{\perp}\right)$. The green curves represent the profiles in Equation (9) with coefficients and uncertainties determined by Equation (12) using $\mathrm{PSD}_{\perp}$ data from $\sim 400$ to $\sim 2500 \mathrm{eV}$. The time periods that the spacecraft was less than $2 \rho$, i.e. $2 \rho / V_{m}$, are also marked in panels (b) - (h), where $\rho$ is energy dependent.

The fitted coefficients are $\beta_{f} \sim 155^{\circ} \pm 2^{\circ}, V_{m} \sim 11.5 \pm 2.2 \mathrm{~km} / \mathrm{s}$, and $t_{0} \sim 6: 10: 47.6 \pm 0.1$ UT for the leading boundary, and $\beta_{f} \sim 336^{\circ} \pm 2^{\circ}, V_{m} \sim 18.1 \pm 3.4 \mathrm{~km} / \mathrm{s}$, and $t_{0} \sim 6: 10: 53.9 \pm 0.1$ UT for the trailing boundary. The uncertainty of $V_{m}$ has been corrected by:

$$
\Delta V_{m}=V_{m} \sqrt{\left(\frac{\Delta^{*} V_{m}}{V_{m}}\right)^{2}+\left(\frac{\Delta \rho}{\rho}\right)^{2}}
$$

where $\Delta^{*} V_{m}$ is the uncertainty obtained from fitting, and $\Delta \rho / \rho(\sim 0.18$ as an upper limit) is the uncertainty due to finite energy resolution.

It is shown that the green curves are in good agreement with the observations in all panels, especially considering the energy dependence of shapes of the curves. The size of the structure is estimated to be approximately $90 \mathrm{~km} \approx 30 \rho_{e}$ (or $0.25 \rho_{i}$ ), where $\rho_{e}$ $\left(\rho_{i}\right)$ is electron (ion) thermal gyro-radius. Note that the difference of $\beta_{f}$ for the leading and the trailing boundaries is very close to $180^{\circ}$. This means the trailing boundary is moving in the same direction as the leading boundary but with a higher velocity $(18.1 \pm 3.4$ $\mathrm{km} / \mathrm{s}$ to $11.5 \pm 2.2 \mathrm{~km} / \mathrm{s}$ ), suggesting that the structure is shrinking.

\section{Discussion}

This paper and Liu et al. (2019) demonstrate that burst mode electron measurements from MMS FPI have been successfully applied for the sounding technique to electron scale structures, which requires high energy, angular, and time resolution as indicated by Equation (4). The sounding technique provides a way to see into the propagation of a trapping boundary based on single-spacecraft observations. We expect this technique to open up new types of analyses for a variety of structures such as electron dissipation region of reconnection, etc. The comprehensive sounding technique proposed in this study takes the motion of the structure into account, which gives a better description of a constant propagation boundary. Coefficients related to the boundary are energy independent, though the fitting is over multiple energy channels. The curve fitting further reduces uncertainty by comprehensively using the data from different energies and times.

This paper gives direct observational evidence showing that the leading and the trailing boundaries of the ESMH are moving in the same direction but with different velocities (Figure 4), suggesting a shrinkage of the structure. The size of the structure may reduce $\sim 36 \%$ (i.e. $(18.1-11.5) / 18.1$ ) during the observation of MMS3. The shrinkage could be the key to the final destiny of the electron scale magnetic cavities, since we expect there to be electric fields induced by the variation of magnetic flux, while a new model is required to show how the structure might evolve.

The coefficients $V_{m}, \beta_{f}$, and $t_{0}$ derived from the comprehensive sounding technique are directly compared to observations from MMS3. An average ion bulk velocity $\left\langle V_{i}\right\rangle$ between 06:10:47.6 and 06:10:53.9 UT is projected to the magnetic perpendicular plane to get $\left|\left\langle V_{i}\right\rangle\right|=13.4 \mathrm{~km} / \mathrm{s}$ and $\beta_{\left\langle V_{i}\right\rangle}=143^{\circ}$, which is close to $V_{m}$ and $\beta_{f}$ determined by the curve fitting. Previously, another structure velocity $V_{M H}$ was evaluated to be $(-5.5,35.5$, $15.0) \mathrm{km} / \mathrm{s}$ in GSE with a mapping analysis applied to the event (Gershman et al., 2016). If projected to the magnetic perpendicular plane, it should be $\left|V_{M H \perp}\right|=10.5 \mathrm{~km} / \mathrm{s}$ and 

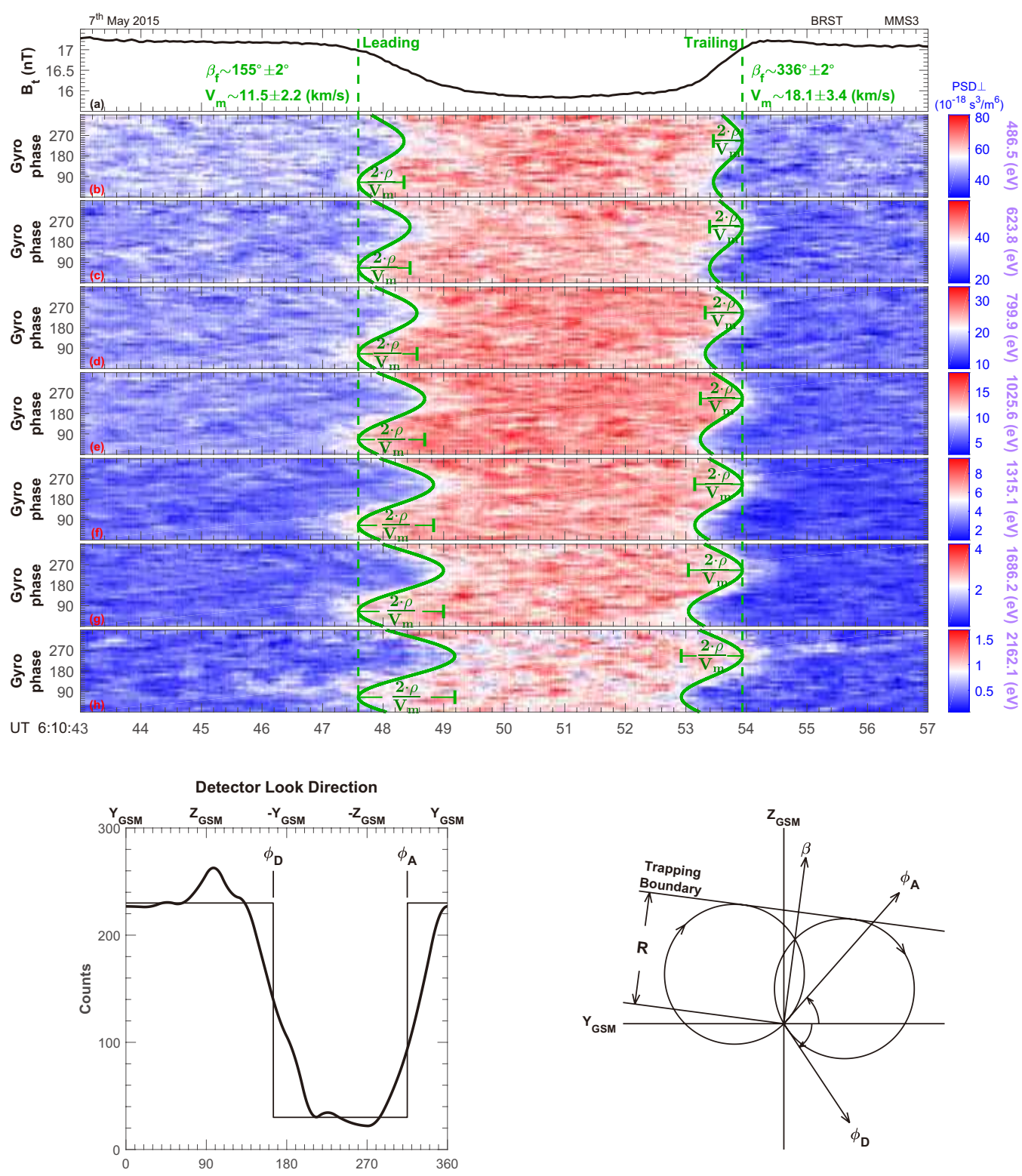

Figure 4. Gyro-phase distributions of perpendicular electron phase space density $\left(\mathrm{PSD}_{\perp}\right)$ for different energy channels from MMS3. In the top part, panel (a) shows the magnetic field strength $B_{t}$, and panels (b) - (h) present $\mathrm{PSD}_{\perp}$ of different energies in the plane perpendicular to the magnetic field, i.e. the XY plane of the newly defined XYZ coordinates. The gyro-phase angle is defined as the angle to the positive $\mathrm{X}$-axis in the XY plane. In each panel, the green curve near the leading or the trailing boundary is from Equation (9). The two vertical dashed lines denote the $t_{0}$ of Equation (9). The boundary orientation $\left(\beta_{f}\right)$ and the boundary moving velocity $\left(V_{m}\right)$ are also labeled near the vertical line with green ink for each boundary. The coefficients $\beta_{f}, V_{m}$, and $t_{0}$ are derived from a curve fitting to $\mathrm{PSD}_{\perp}$ data, detailed in the text. The bottom part of this figure is the same as Figure 1 of Zong et al. (2004) but for GSM coordinates, schematically showing a typical gyro-phase distribution of $\mathrm{PSD}_{\perp}$ at some time near a trapping boundary, which also illustrates the definition of $\phi_{A}$ and $\phi_{D}$ as sharp $\mathrm{PSD}_{\perp}$ declines. 
$\left|\beta_{V_{M H \perp}}\right|=169^{\circ}$, which is also close to $V_{m}$ and $\beta_{f}$. Note that coefficients $t_{0}$ as vertical hashed lines in Figures 1, 3, and 4 have been compared to $B_{t}$ in panel (a) of Figure 4, showing that $t_{0}$ correspond to beginning or ending of the magnetic field decrease. The consistency between fitted coefficients and observations indicates that $V_{m}, \beta_{f}$, and $t_{0}$ are reliable and the comprehensive sounding technique is applicable.

The application of the sounding technique assumes that the boundary is a theoretical trapping boundary that only electrons fulfilling their gyro-motions entirely in the interior can be stably trapped. Later, we confirmed its rationality by a high goodness of the curve fitting shown in Figure 4 (green curves in panels (b) - (h)). This result suggests that the loss of particles via the boundary plays a significant role in forming nongyrotropic distributions of $\mathrm{PSD}_{\perp}$ shown in Figure 2.

The ESMC event discussed in this paper is located in the magnetotail plasma sheet, which was previously studied by Gershman et al. (2016). The sounding technique results in similar scale size but further reveals shrinkage of the structure. Although the characteristics agree with an isolated magnetic mirror, it may have a direct connection to the central plasma sheet due to its large extension in parallel direction (Li et al., 2016; Sundberg et al., 2015; Zhang et al., 2017). Thus, the magnetic field depletion and the electron energization may have an impact on the tail dynamics. Recently, a statistical work has also shown that ESMCs could be sources of a variety of waves in magnetized plasmas (S. T. Yao et al., 2019). Actually, ESMCs could be favorable to the generations of whistler waves via electron temperature anisotropy instabilities (Huang et al., 2019, 2018), indicating that the whistler waves can couple with ESMCs in the turbulent plasmas.

In this paper, we discuss an ESMC event observed by MMS3 on 7 May 2015. A magnetic depression is clearly shown in Figure 1 with simultaneous enhancements of electron fluxes in the local mirror trapping region. The existence of a loss boundary is implied by sky-maps of electron PSDs in Figure 2, and a particle sounding technique is applied to the structure (Figure 3). Then a comprehensive sounding technique is proposed (Equations (5) - (12)) for a boundary with constant moving speed, and applied to the ESMC event discussed in this paper. The result shows that the size of the ESMC is approximately $90 \mathrm{~km}\left(30 \rho_{e}\right)$, i.e. in electron scale; the non-gyrotropic distributions of $\mathrm{PSD}_{\perp}$ is caused by boundary loss, and the velocities and orientations of the leading and the trailing boundaries are obtained (Figure 4). We then suggest that the structure is shrinking according to the evidence that the leading and the trailing boundaries are propagating in almost the same direction but with different velocities, which could be the key to the final understanding of the formation of the structure.

\section{Acknowledgments}

We acknowledge the entire MMS team and instrument leaders for high quality data and convenient access. The FPI data used in this paper can be directly acquired from the MMS Science Data Center (https://lasp.colorado.edu/mms/sdc/public/), and the magnetic field data is publicly available at FigShare (https://figshare.com/s/407404ef903946436718). This work was supported by NSFC 41421003 and NSFC 41627805. H.L. is grateful to financial support from Graduate School of Peking University.

\section{References}

Burch, J., Moore, T., Torbert, R., \& Giles, B. (2016). Magnetospheric multiscale overview and science objectives. Space Science Reviews, 199(1-4), 5-21.

Gershman, D. J., Dorelli, J. C., Viñas, A. F., Avanov, L. A., Gliese, U., Barrie, A. C., ... others (2016). Electron dynamics in a subproton-gyroscale magnetic hole. Geophysical Research Letters, 43(9), 4112-4118.

Goodrich, K. A., Ergun, R. E., \& Stawarz, J. E. (2016). Electric fields associated 
with small-scale magnetic holes in the plasma sheet: Evidence for electron currents. Geophysical Research Letters, 43(12), 6044-6050.

Goodrich, K. A., Ergun, R. E., Wilder, F. D., Burch, J., Torbert, R., Khotyaintsev, Y., ... others (2016). Mms multipoint electric field observations of small-scale magnetic holes. Geophysical Research Letters, 43(12), 5953-5959.

Haynes, C. T., Burgess, D., Camporeale, E., \& Sundberg, T. (2015). Electron vortex magnetic holes: A nonlinear coherent plasma structure. Physics of Plasmas, 22(1), 012309.

Huang, S., Du, J., Sahraoui, F., Yuan, Z., He, J., Zhao, J., ... others (2017). A statistical study of kinetic-size magnetic holes in turbulent magnetosheath: Mms observations. Journal of Geophysical Research: Space Physics, 122(8), $8577-8588$.

Huang, S., He, L., Yuan, Z., Sahraoui, F., Le Contel, O., Deng, X., ... others (2019). Mms observations of kinetic-size magnetic holes in the terrestrial magnetotail plasma sheet. The Astrophysical Journal, 875(2), 113.

Huang, S., Sahraoui, F., Yuan, Z., He, J., Zhao, J., Le Contel, O., ... others (2017). Magnetospheric multiscale observations of electron vortex magnetic hole in the turbulent magnetosheath plasma. The Astrophysical Journal Letters, 836(2), L27.

Huang, S., Sahraoui, F., Yuan, Z., Le Contel, O., Breuillard, H., He, J., .. others (2018). Observations of whistler waves correlated with electron-scale coherent structures in the magnetosheath turbulent plasma. The Astrophysical Journal, $861(1), 29$.

Ji, X.-F., Wang, X.-G., Sun, W.-J., Xiao, C.-J., Shi, Q.-Q., Liu, J., \& Pu, Z.-Y. (2014). Emhd theory and observations of electron solitary waves in magnetotail plasmas. Journal of Geophysical Research: Space Physics, 119(6), 4281-4289.

Kaufmann, R. L., \& Konradi, A. (1973). Speed and thickness of the magnetopause. Journal of Geophysical Research, 78(28), 6549-6568.

Konradi, A., \& Kaufmann, R. L. (1965). Evidence for rapid motion of the outer boundary of the magnetosphere. Journal of Geophysical Research, 70(7), $1627-1637$.

Li, Z.-Y., Sun, W.-J., Wang, X.-G., Shi, Q.-Q., Xiao, C.-J., Pu, Z.-Y., .. Fu, S.-Y. (2016). An emhd soliton model for small-scale magnetic holes in magnetospheric plasmas. Journal of Geophysical Research: Space Physics, 121(5), 4180-4190.

Liu, H., Zong, Q.-G., Zhang, H., Xiao, C. J., Shi, Q. Q., Yao, S. T., .. Rankin, R. (2019). Mms observations of electron scale magnetic cavity embedded in proton scale magnetic cavity. Nature Communications, 10(1040).

Oksavik, K., Fritz, T., Zong, Q.-G., Søraas, F., \& Wilken, B. $\quad$ (2002). Threedimensional energetic ion sounding of the magnetopause using cluster/rapid. Geophysical research letters, 29(9).

Pollock, C., Moore, T., Jacques, A., Burch, J., Gliese, U., Saito, Y., ... others (2016). Fast plasma investigation for magnetospheric multiscale. Space Science Reviews, 199(1-4), 331-406.

Roytershteyn, V., Karimabadi, H., \& Roberts, A. (2015). Generation of magnetic holes in fully kinetic simulations of collisionless turbulence. Phil. Trans. R. Soc. A, 373(2041), 20140151.

Russell, C., Anderson, B., Baumjohann, W., Bromund, K., Dearborn, D., Fischer, D., ... others (2016). The magnetospheric multiscale magnetometers. Space Science Reviews, 199(1-4), 189-256.

Sahraoui, F., Belmont, G., Pinçon, J., Rezeau, L., Balogh, A., Robert, P., \& Cornilleau-Wehrlin, N. (2004). Magnetic turbulent spectra in the magnetosheath: new insights. In Annales geophysicae (Vol. 22, pp. 2283-2288).

Sahraoui, F., Belmont, G., Rezeau, L., Cornilleau-Wehrlin, N., Pinçon, J., \& Balogh, 
A. (2006). Anisotropic turbulent spectra in the terrestrial magnetosheath as seen by the cluster spacecraft. Physical review letters, 96(7), 075002.

Sonnerup, B. Ö., \& Cahill Jr, L. (1967). Magnetopause structure and attitude from explorer 12 observations. Journal of Geophysical Research, 72(1), 171-183.

Sun, W., Shi, Q., Fu, S., Pu, Z., Dunlop, M., Walsh, A., ... others (2012). Cluster and tc-1 observation of magnetic holes in the plasma sheet. In Annales geophysicae (Vol. 30, pp. 583-595).

Sundberg, T., Burgess, D., \& Haynes, C. (2015). Properties and origin of subprotonscale magnetic holes in the terrestrial plasma sheet. Journal of Geophysical Research: Space Physics, 120(4), 2600-2615.

Yao, S., Wang, X., Shi, Q., Pitkänen, T., Hamrin, M., Yao, Z., ... others (2017). Observations of kinetic-size magnetic holes in the magnetosheath. Journal of Geophysical Research: Space Physics, 122(2), 1990-2000.

Yao, S. T., Shi, Q. Q., Li, Z. Y., Wang, X. G., Tian, A. M., Sun, W. J., ... Rème, H. (2016). Propagation of small size magnetic holes in the magnetospheric plasma sheet. Journal of Geophysical Research: Space Physics, 121(6), 55105519. doi: 10.1002/2016JA022741

Yao, S. T., Shi, Q. Q., Yao, Z. H., Li, J. X., Yue, C., Tao, X., ... Giles, B. L. (2019). Waves in kinetic-scale magnetic dips: Mms observations in the magnetosheath. Geophysical Research Letters, 46(2), 523-533. doi: 10.1029/2018GL080696

Zhang, X.-J., Artemyev, A., Angelopoulos, V., \& Horne, R. (2017). Kinetics of subion scale magnetic holes in the near-earth plasma sheet. Journal of Geophysical Research: Space Physics.

Zong, Q.-G., Fritz, T., Spence, H., Oksavik, K., Pu, Z.-Y., Korth, A., \& Daly, P. (2004). Energetic particle sounding of the magnetopause: A contribution by cluster/rapid. Journal of Geophysical Research: Space Physics, 109(A4). 

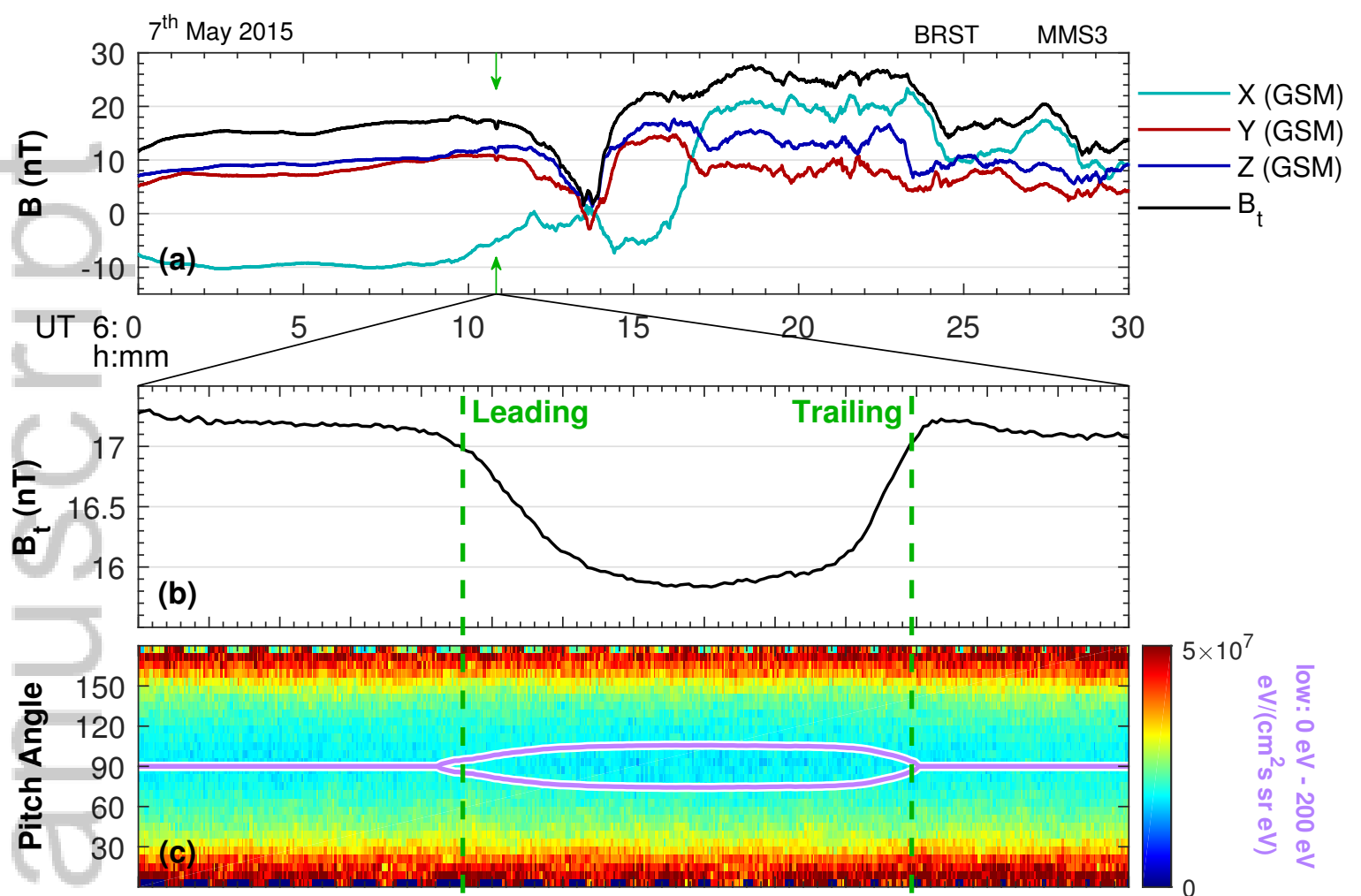

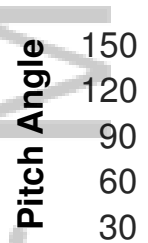
$-$

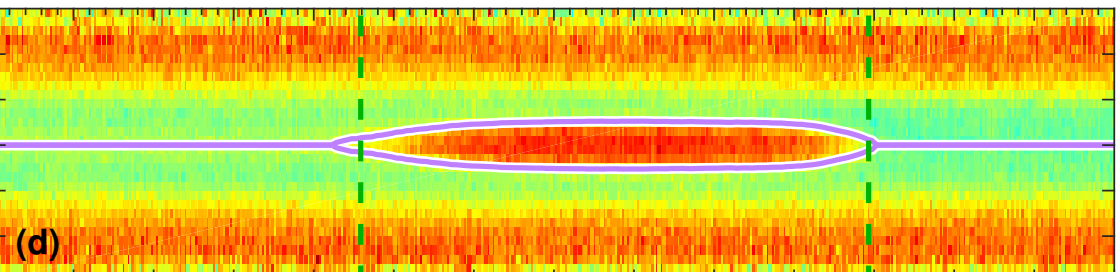

Pा
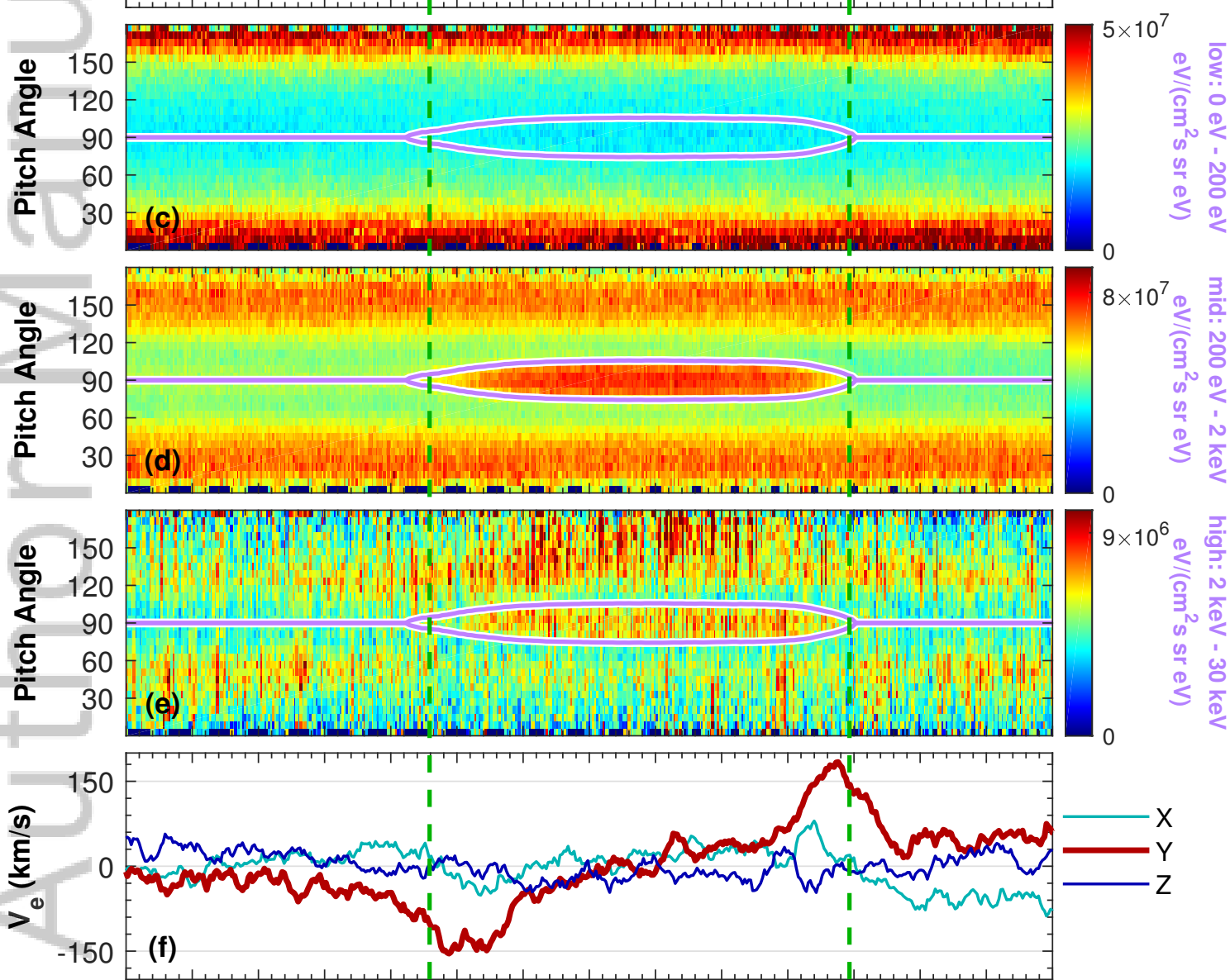

$\begin{array}{lllllllllllllll}\text { UT } 6: 10: 43 & 44 & 45 & 46 & 47 & 48 & 49 & 50 & 51 & 52 & 53 & 54 & 55 & 56 & 57\end{array}$

h:mm:ss L:12 MLT:Thes aMtielte is. protected by copyright. All rights reserved. 
Figure 2.
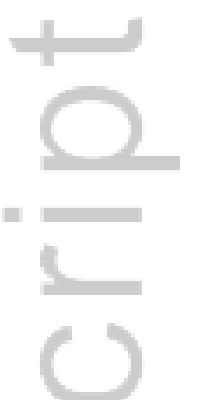

$\infty$
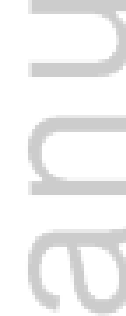

This article is protected by copyright. All rights reserved. 


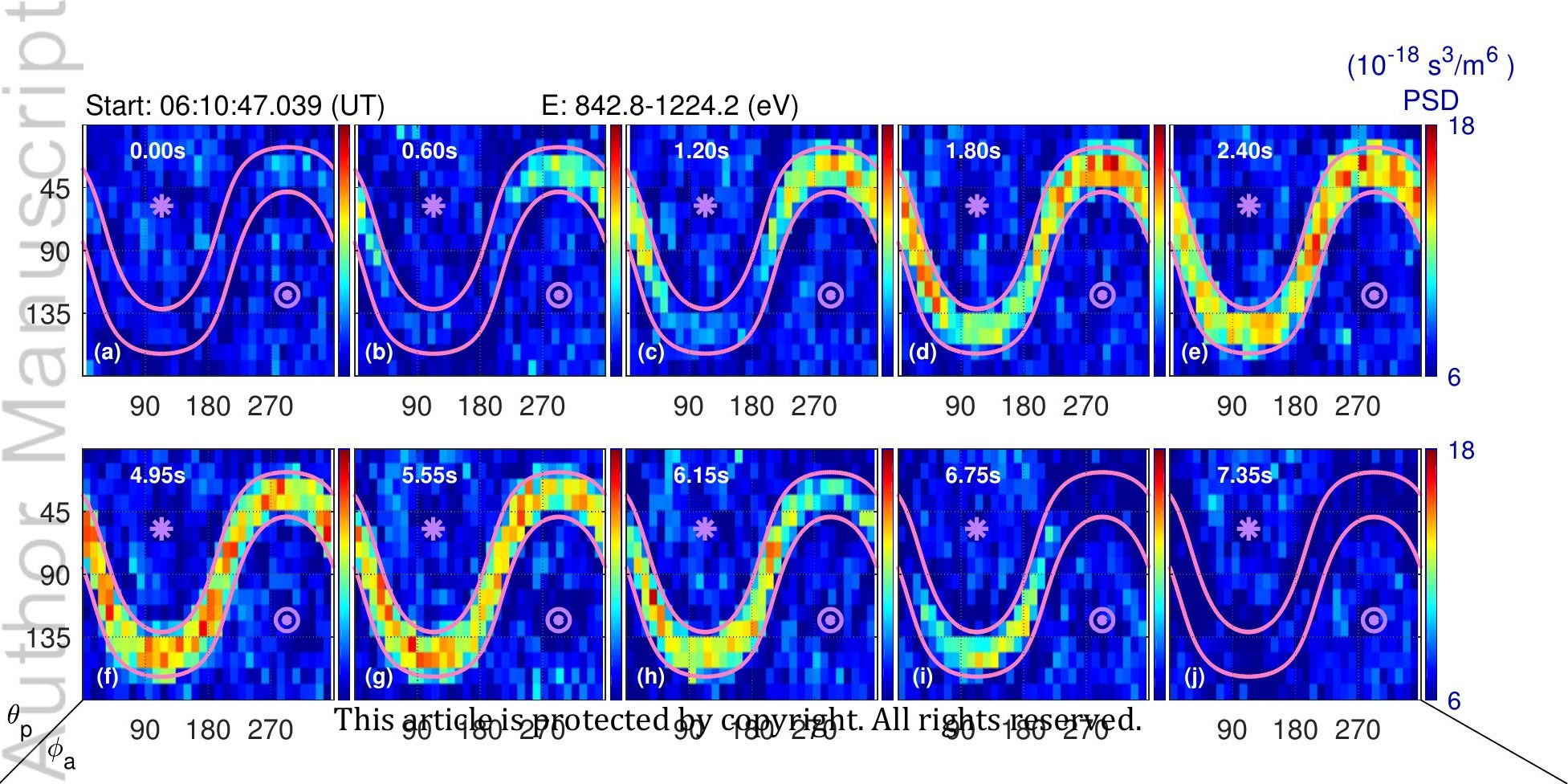



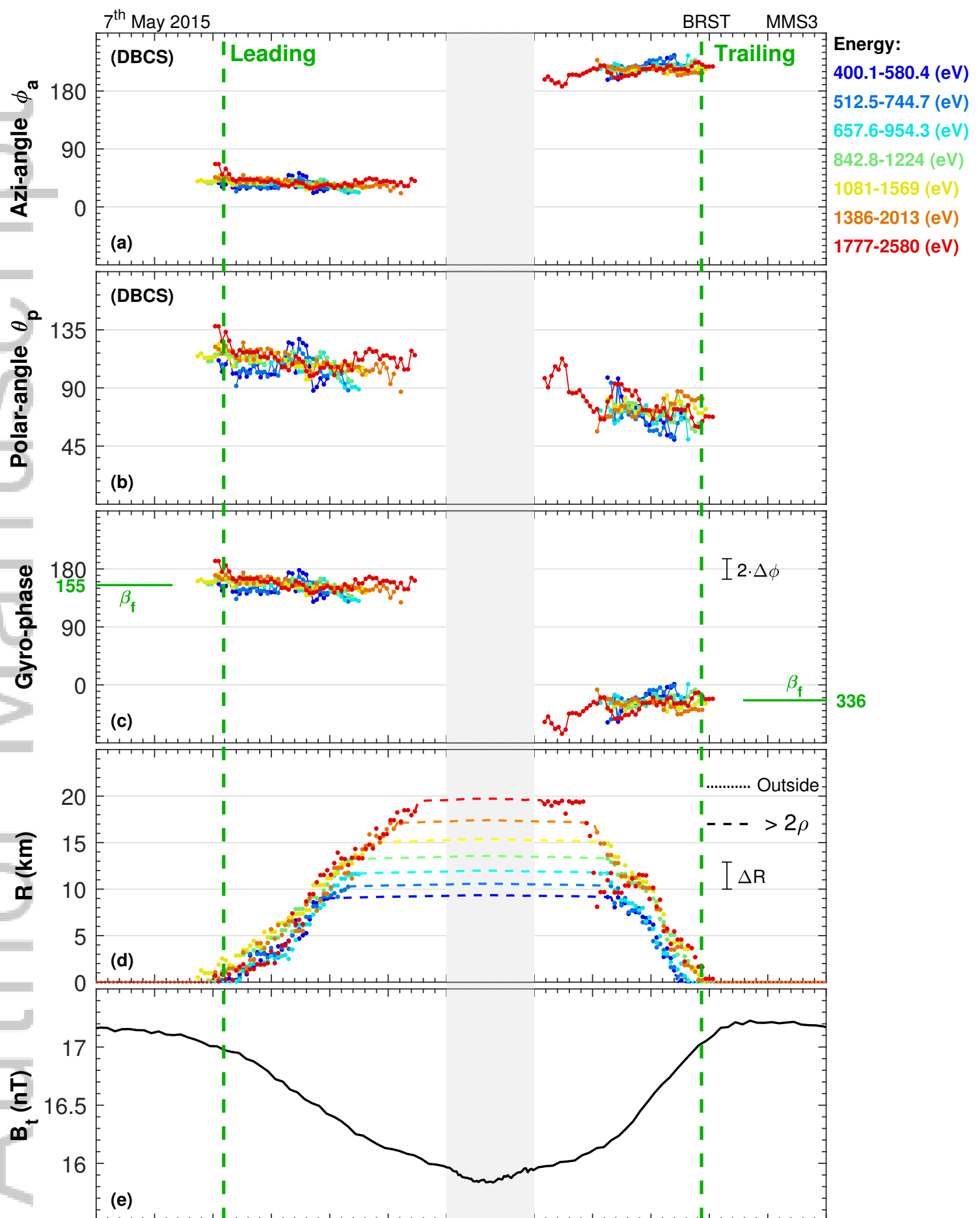

UT 6:10:46.5 $47 \begin{array}{llllllllllll} & 47.5 & 48 & 48.5 & 49 & 49.5 & 52.5 & 53 & 53.5 & 54 & 54.5 & 55\end{array}$

This article is protected by copyright. All rights reserved. 


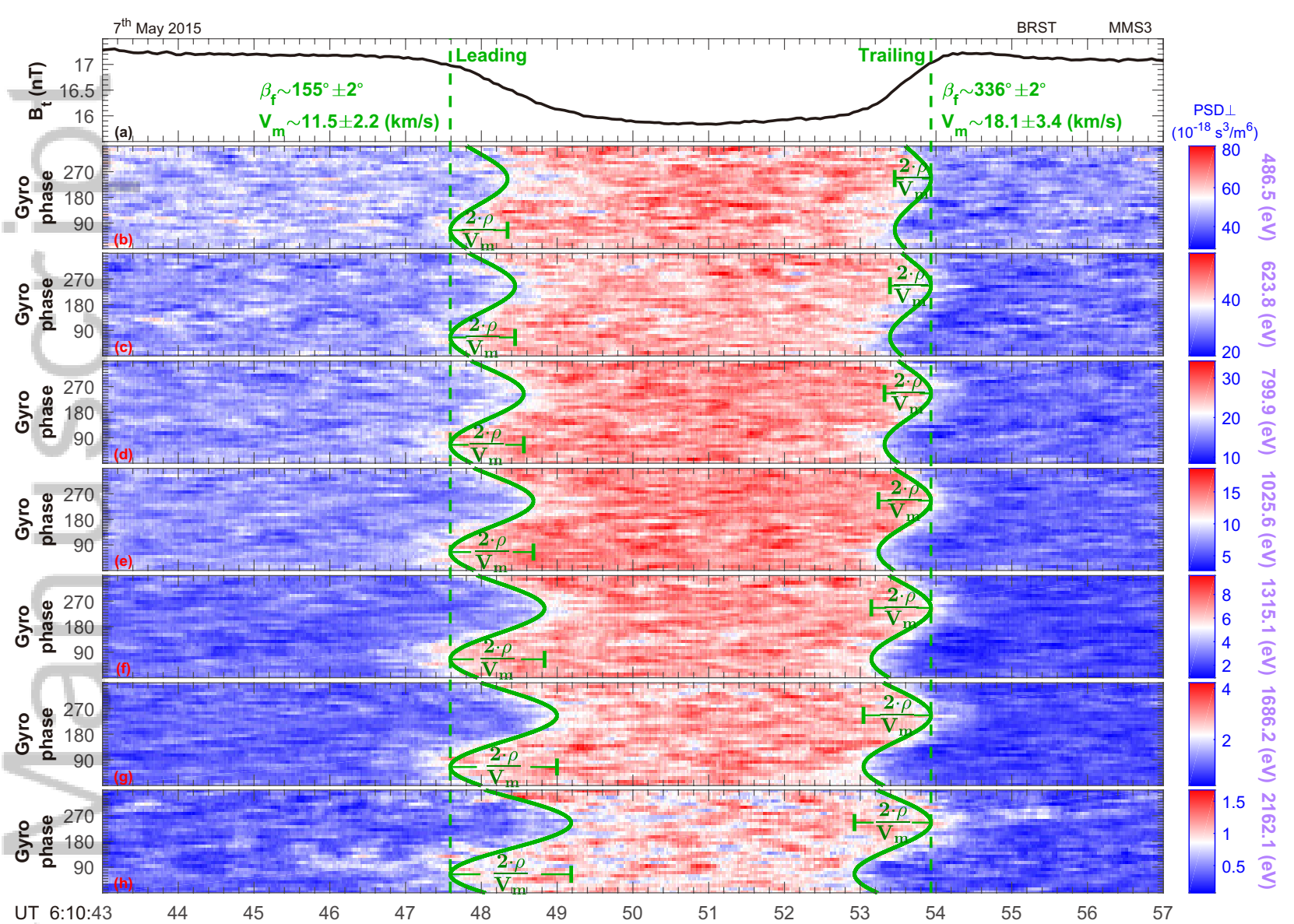

Detector Look Direction

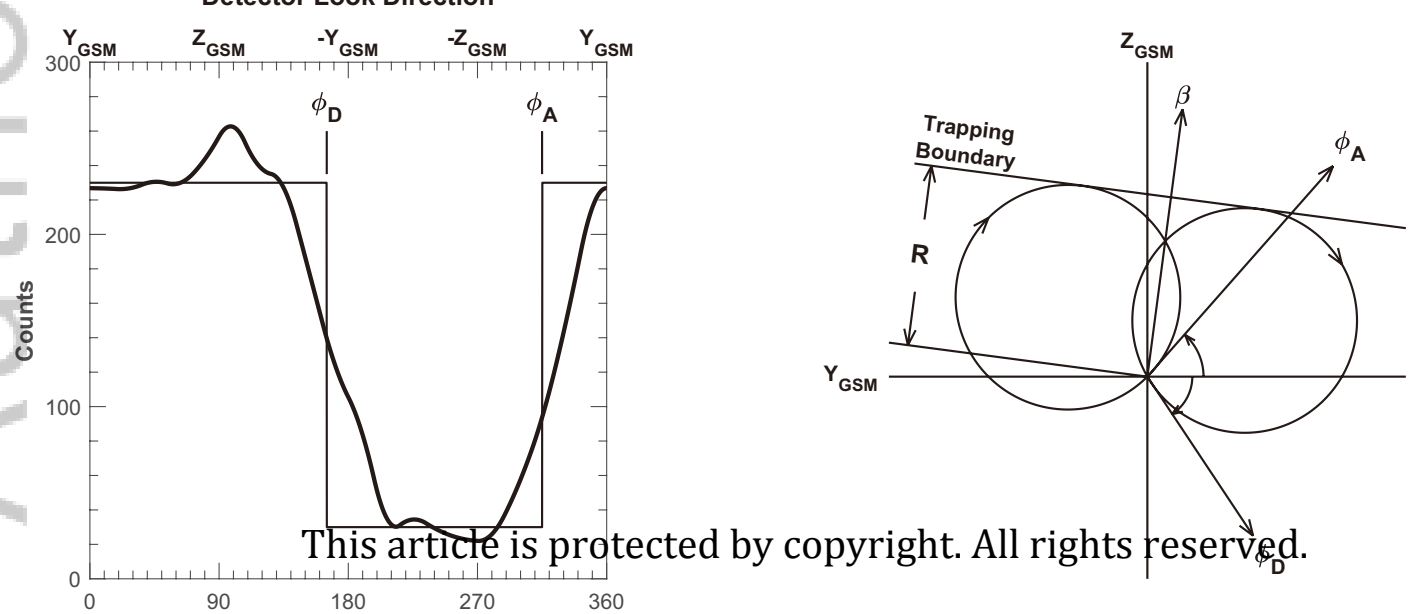



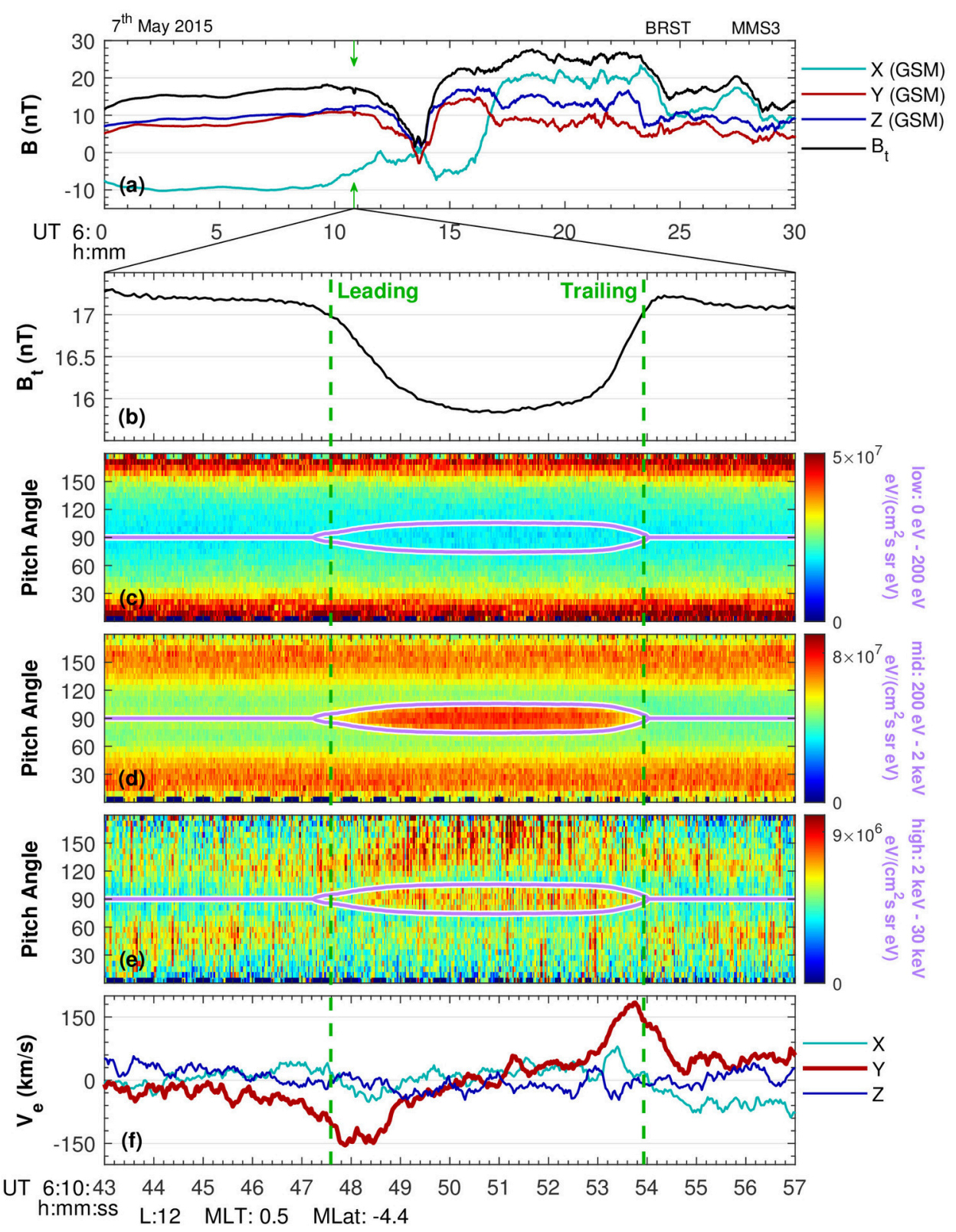

2019gl083569-f01-z-.eps

This article is protected by copyright. All rights reserved. 


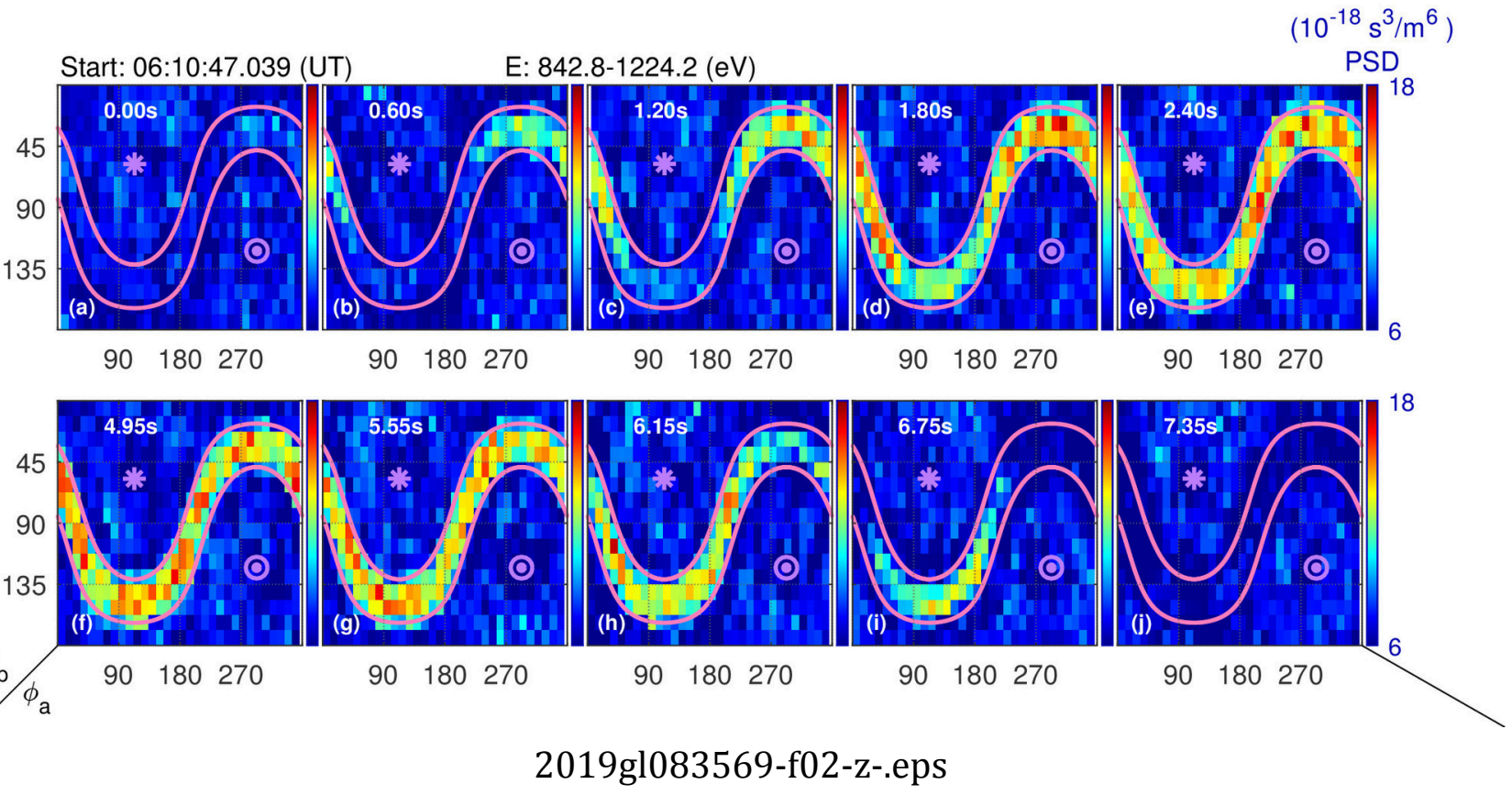

This article is protected by copyright. All rights reserved. 


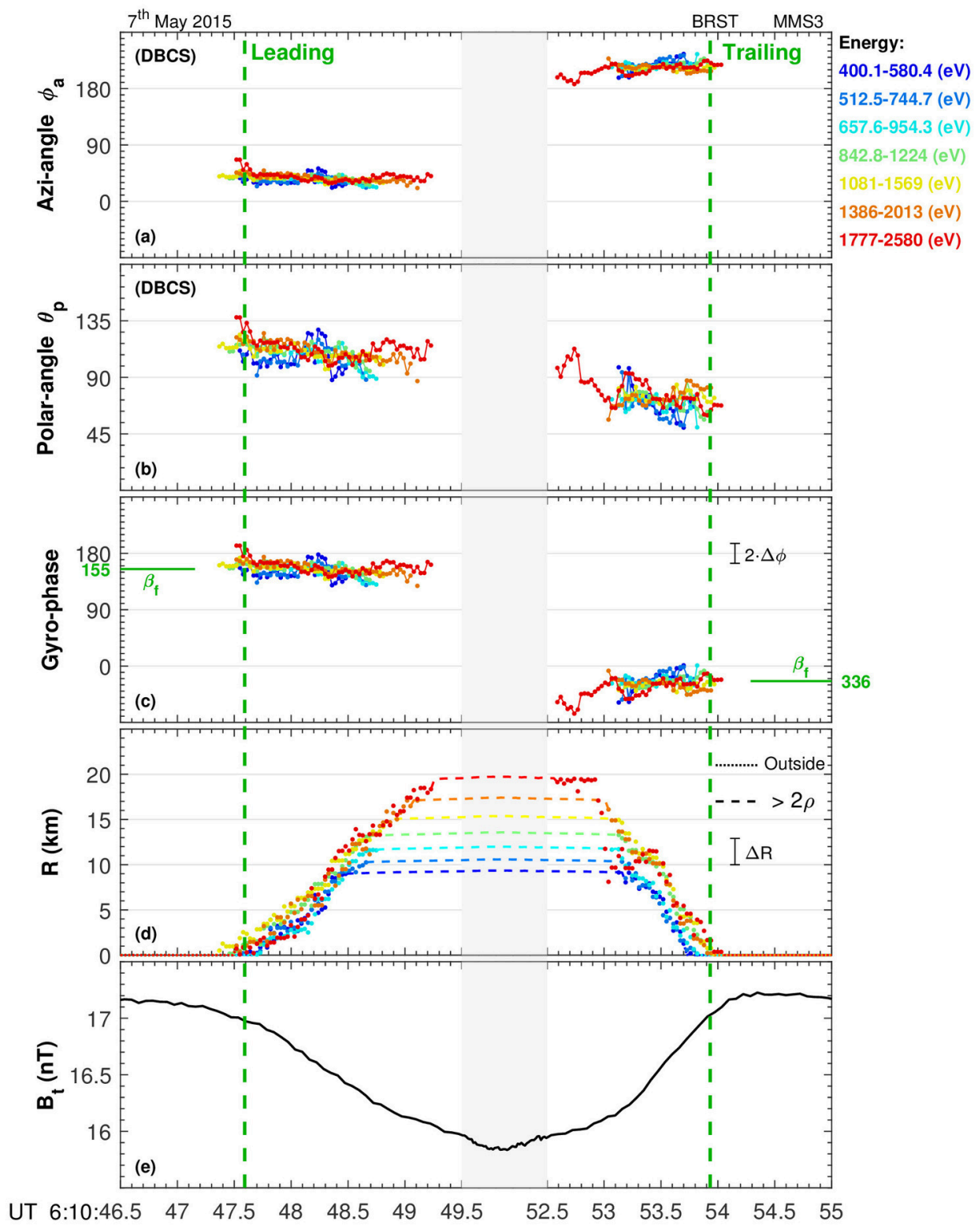

2019gl083569-f03-z-.eps

This article is protected by copyright. All rights reserved. 

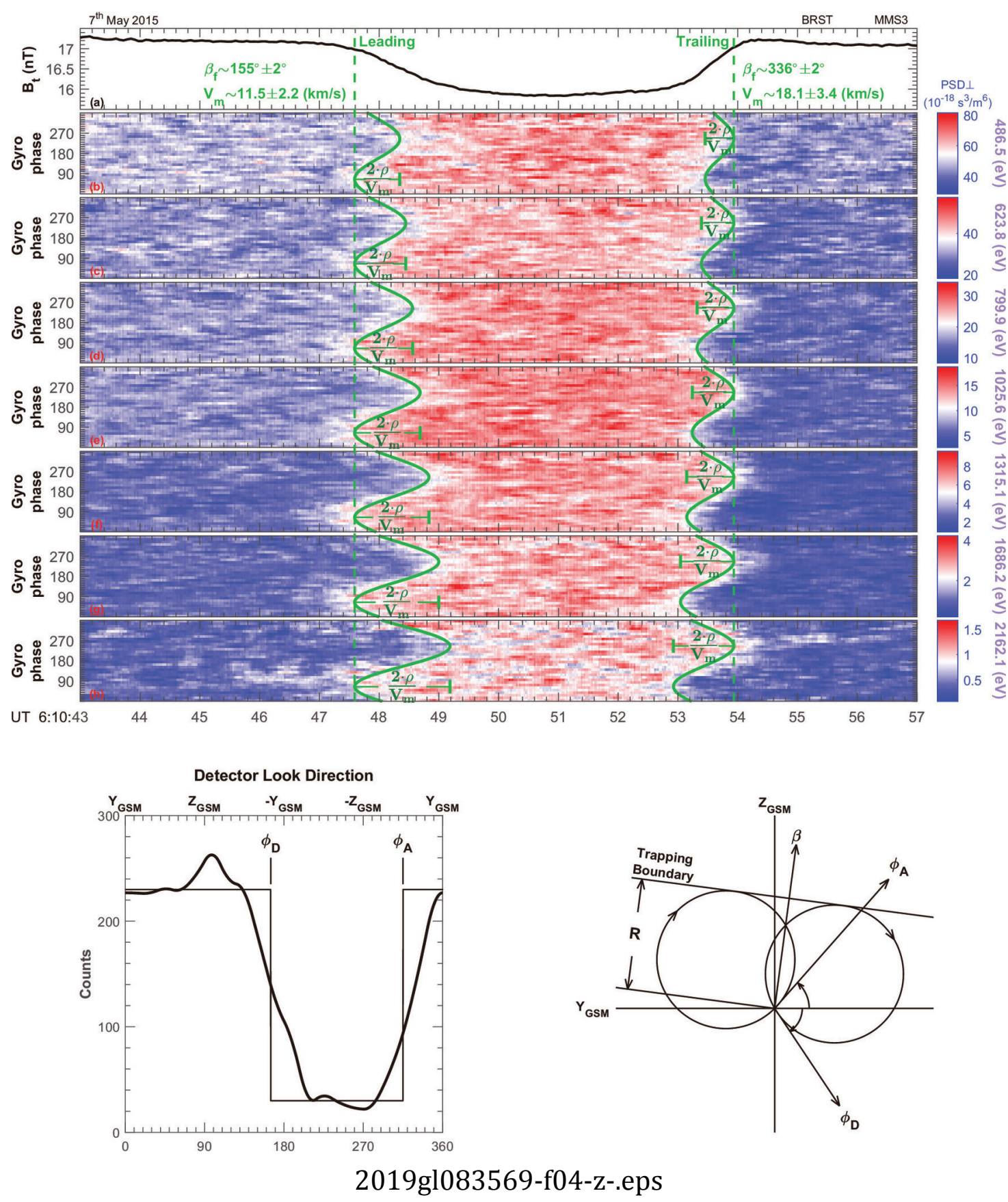

This article is protected by copyright. All rights reserved. 